Note: this is a post-print draft of the journal article:

Dickinson, A.S., Taylor, A.C., Browne, M. (2010) "Performance of the Resurfaced Hip: Part 1The Influence of Prosthesis Size and Positioning on Remodelling and Fracture of the Femoral Neck". Proceedings of the Institution of Mechanical Engineers Part H: Journal of Engineering in Medicine 224(3): 427-439

The final, fully proofed and peer-reviewed journal article is available from the publisher online, via the following link:

http://pih.sagepub.com/content/224/3/427.abstract 


\title{
Performance of the Resurfaced Hip: Part 1- The Influence of Prosthesis Size and Positioning on Remodelling and Fracture of the Femoral Neck
}

\author{
A S Dickinson ${ }^{1}$, A C Taylor ${ }^{2}$, M Browne ${ }^{1}$ \\ ${ }^{1}$ Bioengineering Science Research Group, University of Southampton, Southampton, UK \\ ${ }^{2}$ Finsbury Development Ltd, Leatherhead, Surrey, UK
}

\begin{abstract}
:
Hip Resurfacing is an established treatment for osteoarthritis in young, active patients. Failure modes include femoral neck fracture and prosthesis loosening, which may be associated with medium term bone adaptation, including femoral neck narrowing and densification around the prosthesis stem.

Finite Element modelling was used to indicate the effects of prosthesis sizing and positioning on bone remodelling and fracture strength under a range of normal and traumatic loads, aiming to understand these failure modes better.

The simulations predicted increased superior femoral neck stress shielding in young patients with small prostheses, which required shortening of the femoral neck to give an acceptable implant-bone interface. However with a larger prosthesis, natural femoral head centre recreation in the implanted state was possible, so stress shielding was restricted to the prosthesis interior, and its extent was less sensitive to prosthesis orientation. With valgus orientation, the implanted neck strength was, at worst, within $3 \%$ of its intact strength.
\end{abstract}

The study suggests that femoral neck narrowing may be linked to a reduction in horizontal femoral offset, occurring if the prosthesis is excessively undersized. As such, hip resurfacing should aim to reproduce the natural femoral head centre, and for valgus prosthesis orientation to avoid femoral neck fracture.

Keywords: hip resurfacing, finite element analysis, bone remodelling, neck fracture, prosthesis positioning 


\section{Introduction}

Resurfacing Hip Replacement (RHR) is an established alternative to traditional Total Hip Replacement (THR) for young, active osteoarthritis patients. Resurfacing prostheses offer greater femoral bone conservation to aid revision surgery, reduced dislocation risk, shorter recovery time and, in theory, more natural joint biomechanics, reducing patient perception and adverse bone remodelling effects $[1,2]$. The results of early hip resurfacing surgery showed high levels of aseptic loosening, resulting from osteolytic response to wear particles from the cobalt chromium - polyethylene bearing couple [3, 4]. Since the introduction of metal-on-metal (MoM) resurfacing implants in the late 1990s, the procedure has achieved similar medium term results to THR, despite the demanding young patient cohort [5]. The main failure mode seen in the latest generation of implants is early femoral neck fracture in the first post-operative year, with an incidence of $0.5-2 \%$. There is considerable debate surrounding the cause, and a combination of surgical, patient selection and implant design related factors are thought to contribute [6-11]. As longer follow-up evidence has been collected, narrowing of the femoral neck at the head-neck junction has been identified radiographically in a considerable proportion of resurfacing patients [3, 12-15]. In the most recent studies, narrowing was observed to stabilise after two to three years, and hence the clinical significance is unknown. However, radiographic changes are reported to be more extensive prior to prosthesis migration [14], so a greater understanding of the underlying causes would be beneficial.

In an attempt to find a biomechanical explanation for fracture and narrowing of the femoral neck, the results of several computational stress analysis studies have been published which have made predictions of the stress and strain distribution in the bone supporting femoral resurfacing heads. Early, simplified finite element analysis (FEA) models demonstrated a reduction in stress within the resurfaced femoral head [1618] and biomechanical theory [19] and analysis [20] showed that valgus orientation reduced the risk of femoral neck fracture. Later, more detailed, patient specific models with geometry and materials properties based on Computer Tomography (CT) scans were used to obtain more precise, absolute bone strain predictions [21-30]. These studies have considered the effects of different prosthesis-bone interface conditions, fixation methods including cementless and cemented fixation with different cement mantle thicknesses, and variations in prosthesis positioning including varus-valgus orientation and incomplete 
prosthesis seating. The main findings, in agreement with clinical observations, were that the extent of stress shielding is higher with a fully bonded prosthesis and stem, a larger diameter stem and a thicker cement mantle. Cementless fixation was not found to change the biomechanics compared to those with a cemented implant. However, the effects of varus-valgus orientation have been reported $[25,26,28]$ with contradictory results.

The above studies have not predicted patterns of bone remodelling stimulus which are entirely consistent with clinically observed radiographic changes in the resurfaced hip. While these studies permit a preliminary understanding of the biomechanical behaviour of the resurfaced hip, there are still a number of factors that have yet to be addressed in detail in the literature to date; for example, the effect of clinically relevant implant positioning on the strains in the femur has been the subject of a limited number of studies $[25,28]$. Of increasing significance and relevance to the younger patient population, conventional studies have focussed on gait loading patterns, while the active patient is likely to subject the implant to more traumatic loading regimes such as stumbling and sideways falls.

In the present study therefore, the influence of femoral resurfacing head prosthesis positioning on the biomechanical performance of the joint was investigated. Performance was assessed in terms of the remodelling stimulus under gait loads, and the distribution of failing bone in trauma, for stumbling and oblique falling load cases. 


\section{Methods and Materials}

A subject specific CAD model of the proximal third of the femur was obtained from the computer tomography (CT) scan of a 63 year old male angiograph patient (height $1.77 \mathrm{~m}$, weight $85 \mathrm{~kg}$ ) with no known orthopaedic disorder. The femur geometry was extracted from the CT scan as a mesh using Amira software (Mercury Computer Systems Inc, Chelmsford, MA), and CAD surface geometry was fitted to the anatomy in SolidWorks 2007 software (SolidWorks Corp, Concord, MA). The femoral head was resurfaced with a traditional design hip resurfacing prosthesis, representative of the BHR (Smith\&Nephew, Memphis, TN, USA) and the ADEPT (Finsbury Orthopaedics, Leatherhead, UK) designs.

The prosthesis was positioned according to contemporary operative technique, referencing the maximum diameter of the femoral neck, rather than the femoral head centre. Using SolidWorks, the femur model was partitioned at the prosthesis-bone interface so that it could be analysed first in the intact, preoperative state, and then again with the cut bone removed and the prosthesis introduced. In this way, the mesh in the retained bone would be identical in the pre- and post-operative models, removing averaging errors from the remodelling stimulus calculation. The only deviation of the cut surface of the bone from the prosthesis internal geometry was where the distal section of the traditional design stem tapers, and the drilled bore for the stem was parallel sided and drilled over length by $5 \mathrm{~mm}$.

The biomechanical geometry and free body diagrams in the pelvic and femoral frames of reference are shown in Fig. 1. Two surgical variation metrics were investigated. First, the prosthesis was implanted with $\pm 10^{\circ}$ varus-valgus orientation relative to the neutral femoral neck axis, indicated by the neck-shaft angle (NSA). With the selected $50 \mathrm{~mm}$ bearing diameter prosthesis, this was the maximum variation which could be achieved without notching the femoral neck or leaving exposed cancellous bone at the head-neck junction. However, achieving the desired head neck junction using this small sized prosthesis resulted in reduction of the horizontal femoral offset and the abductor muscle moment arms (HFO and AMA, Fig. 1), as shown by the data in Table 1. A coordinate system was created, aligning the mechanical axis of the femur with vertical (Fig. 1, right). The mechanical axis-shaft angle (MASA) was adjusted where the prosthesis position changed the femoral head centre by tilting the bone in the frontal plane, so that the mechanical axis remained vertical (Table 1). This is based on the assumption of a constant femoral 
equilibrium force (FEF) and femoral equilibrium moment (FEM) applied to the bone by the other muscles and bone structures. To isolate the effect of femoral offset from that of varus-valgus positioning, a second group of models was produced by resurfacing with a $52 \mathrm{~mm}$ bearing diameter prosthesis, which permitted the natural femoral head centre position and horizontal offset distance to be recreated in the resurfaced joint, throughout the range of varus-valgus angles.

The bone and prosthesis geometry was imported into ANSYS V11 (ANSYS Inc, Canonsburg, PA) FEA software where it was meshed with second order solid elements: tetrahedral in the bone, and mapped hexahedra in the prosthesis. The implanted mesh contained approximately 160,000 nodes, refined in the proximal bone and at the prosthesis-bone interface. The bone elements were assigned linear isotropic materials properties using Bonemat software (Rizzoli Institute, Bologna, Italy) referring to the original CT data and linking the bone Young's Modulus to the bone density using the relationship E $=6950 \rho^{1.49}[31]$. The prosthesis elements were assigned a Young's Modulus of 200GPa representing Cobalt Chromium, and a region of elements approximately $2.5 \mathrm{~mm}$ thick in the bone at the interface with the prosthesis were set at 2.8GPa, representing PMMA bone cement interdigitated bone [22]. The stem was modelled in sliding contact with the bone with a nominal friction coefficient of 0.4 [22]. The mesh was verified using a convergence analysis with the distribution of Young's Modulus, strain and strain energy density (SED) throughout the bone as convergence criteria.

Three load cases were modelled, representing one gait and two traumatic scenarios. First, the femoral neck fracture scenario was analysed in stumbling, by applying a joint contact force (JCF) to the femoral head at $8^{\circ}$ adduction representing the worst case loading condition identified from a cadaver study [32]. The joint contact force was applied using a circular pressure distribution over a circular patch of nodes on the bearing surface according to Hertzian theory [33] and corroborated by Udofia et al [34], of 5mm radius for the implanted cases and an approximated $10 \mathrm{~mm}$ radius for the intact case. Second, femoral neck fracture was investigated for 'oblique' falling, in a sideways and backwards direction, also studied previously for the intact hip [35-37]. The femur was oriented with the femoral shaft inclined at $30^{\circ}$ to horizontal and the femoral neck angled $20^{\circ}$ anteriorly, and the joint contact force applied to the femoral head, and reacted at the greater trochanter. Finally, a gait scenario was modelled representing normal walking, simulated by $2.0 \mathrm{kN}$ (or $2.4 \mathrm{x}$ bodyweight) joint contact force on the femoral head with $13^{\circ}$ 
adduction and $8^{\circ}$ flexion [38], and a $0.87 \mathrm{kN}$ (or 1 x bodyweight) abductor muscle force (AMF) at $33^{\circ}$ to vertical in the frontal plane, directed towards the centroid of the gluteus muscle origins and wrapping points on the pelvis.

The femoral neck fracture risk was assessed quantitatively by calculating a risk factor (RF) for each element [36, 39-42], as the ratio of the greater of its tensile and compressive principal strains to a tensile or compressive yield strain from in-vitro data [43]. In tension, yield strains of cancellous and cortical bone were set at $6200 \mu \varepsilon$ and $7300 \mu \varepsilon$ respectively. For compression, a yield strain of $10400 \mu \varepsilon$ was used. To compare the performance of the models, loading was increased in $0.05 \mathrm{kN}$ increments and RF was calculated, and the load of the first failing element recorded as the predicted femoral neck fracture load.

The remodelling stimulus was quantified using strain energy density (SED) based methods [44, 45], whereby the percentage change in SED was calculated from the pre- to post-operative conditions. A threshold level of remodelling stimulus of $\pm 75 \%$ change was used in accordance with previous studies [23, 28]. This value was an empirical fit of FE model results to radiographic data from older, total hip arthroplasty patients [44] and resulted in relatively small volumes of remodelling bone, so a second threshold value of $\pm 50 \%$ change was used, to represent the younger patient with a more active metabolismthe target patient for hip resurfacing. 


\section{Results}

Results are presented in two sections:

- the femoral neck fracture risk in traumatic loading, and

- the bone remodelling stimulus in gait loading.

\subsection{Femoral Neck Fracture Risk}

The femoral neck fracture load was predicted for the bone resurfaced with the $50 \mathrm{~mm}$ and $52 \mathrm{~mm}$ prostheses in varus, neutral and valgus orientations and compared to the natural bone, for stumbling and sideways falling loads. The results are included in Fig. 3 and Fig. 4, with the distribution of failing elements in Fig. 5 for a given load: $6 \mathrm{kN}$ in stumbling and $3 \mathrm{kN}$ in falling.

Under stumbling loading, the results predicted that resurfacing the femoral head with the smaller, 50mm prosthesis would reduce the femoral neck fracture risk, giving a higher predicted femoral neck fracture load. Compared to the natural case, the fracture load was predicted to increase by approximately $8 \%, 9 \%$ and $18 \%$ for varus, neutral and valgus orientations respectively. When the $52 \mathrm{~mm}$ prosthesis was used, and the natural femoral head centre was recreated postoperatively, the femoral neck fracture risk was increased by resurfacing, reducing the predicted fracture load by approximately $20 \%$ with varus implant orientation and $9 \%$ in neutral orientation. In valgus orientation, the predicted neck fracture load was within $2 \%$ of the intact case.

The results obtained from the $52 \mathrm{~mm}$ prosthesis models (without any confounding effects of femoral offset) showed a positive correlation between the femoral neck fracture load and increasing valgus prosthesis orientation. This may be explained by the increased proportion of load which is transferred to the femoral neck in compression when the prosthesis is valgus oriented, reducing bending stresses and shear at the head-neck junction $[19,20]$. It is also illustrated by analysis of the location of damage initiation, as seen in Fig. 5. In the valgus case, where the predicted neck fracture load was within $2 \%$ of the natural case, the damage initiated in the superior femoral neck, in the same location as the natural case. However, with the neutral and varus oriented prostheses, the first damage initiated in the bore for the stem of the prosthesis, 
and the reduced predicted fracture loads suggest that the femoral neck would be weakened in these cases. Fig. 5 does not contain results for the $50 \mathrm{~mm}$ prosthesis because the damage initiation stumbling load was greater than $6 \mathrm{kN}$ in all cases.

The same trend of reduced neck strength with increasing varus prosthesis orientation was seen for the smaller, $50 \mathrm{~mm}$ prosthesis as with the $52 \mathrm{~mm}$ resurfacing head, but the femoral neck fracture load was higher for all the femurs resurfaced with the smaller prosthesis. Neck strength was highest when the femoral offset was reduced most, with valgus orientation of the $50 \mathrm{~mm}$ prosthesis, and this may be explained by the reduced femoral offset which generates a lower bending moment on the femoral neck. The applied load was sustained to a greater extent as compression in the femoral shaft, resulting in higher femoral neck fracture strength.

For sideways falling, all fractures were predicted to originate from the anterior-medial femoral neck, as seen in Fig. 5. A similar improvement in neck fracture strength with smaller prosthesis size and therefore reduced femoral offset can be identified in the results for sideways falling, as seen in Fig. 4, where the strength was higher for the hips resurfaced with the $50 \mathrm{~mm}$ head, by up to $12 \%$ for neutral orientation. However, the proximity of the prosthesis stem bore to the medial femoral neck when the prosthesis was oriented in valgus resulted in lower fracture strength than the other orientations, despite its lower offset, but all strength values were above that for the intact bone. The neck strength was lower for the larger prosthesis, but within $3 \%$ of the natural strength for all orientations. Again, Fig. 5 does not contain results for the $50 \mathrm{~mm}$ prosthesis because the damage initiation falling load was greater than $3 \mathrm{kN}$ in all cases 


\subsection{Bone Remodelling Stimulus}

The remodelling stimulus (percentage change in strain energy density) was calculated for the six implanted cases and is shown for a cross section along the femoral neck axis in Fig. 6. This indicates the locations in which bone resorption and densification would be expected; for an elderly patient these correspond to a stimulus below $-75 \%$ and above $75 \%$ respectively, shown by the bottom and top contours on the charts. In all cases, extensive stress shielding was predicted within the superior femoral head, with densification around the stem bore, particularly around the narrowest point of the femoral neck, and at the tip of the stem bore. Apart from a small region in the inferior side of the stem bore, stress shielding was restricted to the interior of the femoral head for the elderly patient.

However, if a threshold remodelling stimulus of 50\% was assumed, for a younger patient, stress shielding and bone densification are indicated by the bottom and top two contours. In that case, stress shielding was predicted to extend into the superior femoral neck when the smaller, $50 \mathrm{~mm}$ prosthesis was used, for all orientations.

Fig. 7 quantifies the relative extents of bone remodelling for the six models, containing charts showing the volume of bone which would be stress shielded and in hypertrophy for each of the six cases, for an elderly patient ( $\pm 75 \%$ threshold remodelling stimulus) and for a younger patient with a more reactive metabolism ( $\pm 50 \%$ threshold stimulus). These charts predict a trend for increased remodelling as the varus-

valgus angle of the prosthesis increases. The volume of hypertrophic bone was predicted to be similar for both sizes and all orientations, but the results suggested that stress shielding would increase with valgus positioning, particularly for the smaller $(50 \mathrm{~mm})$ prosthesis and the younger patient. The volume of bone at the extreme magnitudes of remodelling stimulus ( $\pm 75 \%$ threshold) was between $7.5 \%$ and $8.1 \%$ for the $50 \mathrm{~mm}$ prosthesis, of which $6.5 \%$ to $7.0 \%$ represented stress shielding. There was a slight increase in stress shielding with increasing valgus orientation, and a hip resurfaced with the $52 \mathrm{~mm}$ prosthesis was predicted to follow a similar trend with $6.9 \%-8.1 \%$ of the proximal bone stress shielded. However, the volume of densifying bone was predicted to be higher for the larger prosthesis, in particular in the inferior femoral head and at the tip of the stem bore. 
Analysis of the volume of bone with a remodelling stimulus greater than the $\pm 50 \%$ thresholdrepresenting the younger patient- indicated that stress shielding in particular would be more severe if a small prosthesis were used, oriented in valgus. This is evident in Fig. 7. From Table 1, it may be hypothesised that this increase in stress shielding results from the reduction of the cantilever length and therefore the bending moment on the femoral neck as a result of the shortened femoral offset measurement. Analysis of the remodelling stimulus distribution charts in Fig. 6 shows that the extent of stress shielding inside the femoral head was similar for all prosthesis orientations, and that this increased remodelling stimulus arose instead from increased stress shielding in the superior femoral neck, which would be consistent with a reduced femoral neck bending moment. This theory is further supported by the fact that the volume of stress shielded bone was predicted to be considerably lower and almost unaffected by prosthesis varus-valgus orientation for the younger patient if the $52 \mathrm{~mm}$ prosthesis were used. In that case, the natural joint centre (and therefore the femoral offset) could be recreated postoperatively. In this scenario, irrespective of prosthesis orientation, stress shielding was not predicted to extend into the femoral neck. This trend of increased stress shielding with reduced femoral offset is illustrated in Fig. 8. 


\section{Discussion}

Despite excellent medium term clinical results, there is scope for improvement of resurfacing hip prosthesis surgery to reduce the incidence of early femoral neck fractures, and to prevent longer term bone adaptation which may lead to later neck fractures or prosthesis loosening. Whilst patient selection and education play a major role in the outcome, the biomechanics of the resurfaced hip joint have a considerable effect and were investigated in this study using an FE model, considering the effects of a range of surgical orientations and two prosthesis sizes on the femoral neck fracture risk and the extent and pattern of bone remodelling in the femoral head and neck.

The first part of the study looked at the effects of prosthesis positioning and sizing upon the femoral neck fracture load under stumbling and sideways falling scenarios. In stumbling, the natural femoral neck fracture strength was predicted to be recreated in the resurfaced joint if the prosthesis was oriented with $10^{\circ}$ of valgus compared to the neck axis, with its bearing centre at the natural joint centre location. This was achieved with a $52 \mathrm{~mm}$ prosthesis. Neck fracture strength was predicted to decrease approximately linearly as the prosthesis angle moved through neutral to $10^{\circ}$ of relative varus orientation. The results are in close agreement with clinical experience which has identified excessive varus orientation as a risk factor $[8,20]$. The results are also in agreement with in-vitro mechanical test results, such as the study of resurfaced synthetic and cadaveric femurs by Davis et al [46], which also identified an approximately linear correlation between neck strength and varus-valgus angle, and recreated natural fracture strength with a prosthesis at around $10^{\circ}$ of valgus orientation.

Prosthesis positioning with a reduction in the horizontal femoral offset distance was necessary when the smallest possible (50mm prosthesis) was used, in order to avoid notching the femoral neck. This increased the femoral neck fracture load for all prosthesis orientations. Whilst a reduction in femoral offset distance would, during gait, result in increased abductor muscle force and therefore joint contact force, under instantaneous traumatic events without muscle involvement such as those modelled in this study, this femoral neck cantilever length effect dominates, so the shortened femoral neck made the resurfaced joint stronger. The predicted fracture origin in the natural bone, the superior femoral neck, was representative of one of the morphologies identified by Cristofolini et al's cadaveric tests [32] using the same loading 
conditions, and the fracture origin only moved from this region when the prosthesis was oriented and sized to weaken the femoral neck. In this case, damage initiated in the bore for the prosthesis stem around the narrowest point of the femoral neck, and could reasonably propagate across to the prosthesis rim. This would be in agreement with the implanted fracture morphologies identified by Morlock et al's retrieval analysis [47].

The model indicated that under loads representing a sideways fall, the femoral neck bone damage initiation load was increased by up to $10 \%$ when the femoral offset was shortened. In this case, the damage initiation load was predicted to be lowest, and the same as the intact bone, for the valgus oriented prosthesis, because this is the position in which the stem bore is closest to the damage initiation site at the medial femoral neck, as seen in Fig. 5. The femoral neck fracture risk was very similar to the intact case when the hip was implanted with the larger prosthesis (within 3\% of the fracture load), so considering the degree of uncertainty associated with the FEA modelling approach, it can be concluded that in a sideways fall onto the greater trochanter, there may be no significant increase in the femoral neck fracture risk following resurfacing.

The second part of the study was concerned with the effects of prosthesis varus-valgus orientation and prosthesis sizing, and therefore femoral offset, upon the remodelling occurring in the supporting femoral head and neck bone. Patterns of bone remodelling based on the immediate post-operative remodelling stimulus were predicted to be in agreement with previous modelling studies and clinical observations. This included bone resorption in the superior femoral head, observed in loosened implants [16-18, 20-29], and densification to form sclerotic 'pedestal lines' around the prosthesis stem tip and along its superior edge $[14,48]$. These were predicted to occur for both prosthesis sizes and all implant orientations. Previous modelling studies have linked these pedestal lines to stem tip load transfer [17, 24], although in the immediate post-operative state there is no contact between the stem tip and the bone, and these results agree with more recent modelling results by Ong et al [28] which indicated that the presence of the bore alone is sufficient to cause bone densification around the stem.

Previous studies have investigated the effects of varus-valgus orientation on the bone remodelling stimulus, with modelling studies obtaining contradictory results. Long and Bartel [25] found that the 
prosthesis had to be displaced by $5 \mathrm{~mm}$ distally along the femoral neck axis from the natural head centre in order to avoid exposure of reamed cancellous bone; this resulted in very similar reductions in femoral offset to this study. They reported an increased risk of femoral component loosening for the varus orientation, but only by consideration of cement peak tensile stress. They also reported the load transfer across the implant-bone interface and indentified simply that the loading of the dense femoral head cancellous bone was reduced for all implanted cases. Radcliffe and Taylor [26] used a remodelling stimulus approach, and identified a more natural strain distribution in the femoral neck for a valgus oriented prosthesis. They investigated varus-valgus angles of $\pm 10^{\circ}$ but they sized the prosthesis sufficiently that the natural femoral head centre was recreated for all orientations. They also over-reamed the stem bore, and modelled the ASR prosthesis (DePuy International) which has a fully tapering stem: as such, there would be no remodelling effects of the prosthesis stem, only of the bore drilled to accommodate it. Conversely, the results obtained by Ong et al [28] from $\pm 15^{\circ}$ of varus-valgus orientation indicated a considerable increase in femoral head stress shielding and the extension of stress shielding into the superior femoral neck for the valgus prosthesis. They modelled the same design prosthesis as in this study, giving stem-bone contact along its cylindrical portion, just beyond the rim of the prosthesis. Furthermore, instead of oversizing the prosthesis to maintain the natural head centre, their models appeared to be more representative of clinical results, simulating a similar reduction in horizontal femoral offset with valgus prosthesis positioning.

Clinical data on hip resurfacing has enabled an understanding of the effects of prosthesis positioning and sizing on bone remodelling. Silva et al [49] reported in their study of 50 RHR patients that the pre- to postoperative horizontal offset was reduced by an average of $0.8 \mathrm{~mm}(\mathrm{p}=0.21)$ but that osteoarthritic hips had a significantly reduced horizontal femoral offset compared to the contralateral joint, by an average of $8.4 \mathrm{~mm}(\mathrm{p}<0.00001)$. They identified that this horizontal offset decrease resulted largely from a shift of the natural neck-shaft angle into valgus, as an osteoarthritic deformity. However, all the operations were conducted by a highly experienced surgeon from a centre of excellence, so this may represent the best case of prosthesis positioning. Considering the clinical data on femoral neck narrowing, Hing et al [13] studied 163 hips, also resurfaced by experienced surgeons, and identified the female gender and more valgus natural femoral neck-shaft angle as statistically significantly increased risk factors for neck narrowing. 
They also identified a slightly increased risk of neck narrowing where a smaller prosthesis head size was used. Similarly, Amstutz et al [50] reported on 400 patients and identified a statistically significantly increased risk of femoral neck radiographic changes and femoral component loosening with smaller prosthesis size in males.

Stress shielding is only one potential cause of narrowing of the femoral neck. Other previously suggested causes include inflammatory response to wear particles, impingement, and bone necrosis, possibly caused by exothermic bone cement polymerisation or disruption of the blood supply to the femoral head, specifically the retinacular vessels $[11,13,15,51]$. Considering its high reported incidence and the extreme biomechanical conditions that this modelling study suggests are required for it to occur, it is probable that a combination of these factors is required if narrowing of the femoral neck is to be observed. Spencer et al [15] report that neck narrowing occurs early and stabilises around two years postoperatively; therefore, stress shielding could be the most significant factor. The present model suggests how that factor, at least, may be avoided. If the surgeon aims to preserve the pre-operative horizontal femoral offset, the change in strain pattern in the femoral neck compared to the natural joint may be reduced. This could prevent narrowing of the femoral neck which, if extensive, may lead to loosening. However, provided the surgeon attempts to orient the prosthesis in valgus with respect to the femoral neck axis, this would still avoid weakening the femoral neck, which is the greatest short term concern.

The results of all computational modelling studies must be analysed with consideration of the limitations of the modelling techniques and simplifications. Standard verification checks were made during the modelling process, including comparison of the model's global displacements and strains to clinical data $[52,53]$ and previous modelling studies $[54,55]$, and a finite element mesh convergence study. Non-linear frictional contact was defined in the model, and the peak contact penetration was the order of $10^{-3} \mathrm{~mm}$, or 1000 times less than the contact element size, which was considered acceptable. As discussed previously, comparisons were made where possible between the model's predictions and clinical observations, in order to give confidence in the conclusions drawn. 
However, the model is still subject to several simplifications which must be noted. First, the model simulations were carried out on a model of a single femur. The model was generated from a CT scan of an ideal candidate, male patient, receiving a prosthesis from the most common size range [5]. To remove any effects of orthopaedic degeneration of the joint which would certainly be present, but subject to a very large range of variability, and to isolate the effects of prosthesis positioning, a disease-free candidate was selected from 15 available scans. This was considered to be reasonable because the effects of conditions such as sclerosis or destruction of the subchondral bone would be largely removed by the surgical cuts, and the isolated femur modelling method avoids the effects of narrowing of the joint space. Other effects such as formation of osteophytes and subchondral cysts, or avascular necrosis would be subject to greatest variability, but, if advanced, may be included in the list of contraindications for hip resurfacing. Therefore, this ideal candidate, disease-free patient was used for the same reasons that the investigated range of surgical variability was restricted to avoid surgical error scenarios as incomplete prosthesis seating, notching of the femoral neck, and excessive cement penetration: to allow reliable comparison of the variables of interest. The limitation of a single bone analysis is that results should be used to investigate the effects of surgical positioning and sizing variables in comparative quantitative terms rather than making absolute predictions, which was the approach taken in this study.

One simplification of the model boundary conditions concerns the fact that the same joint contact force (JCF) and abductor muscle force (AMF) were applied to the model irrespective of the reduction in horizontal femoral offset (HFO) and abductor moment arm (AMA) of the joint which resulted from resurfacing with the smaller, $50 \mathrm{~mm}$ prosthesis. The forces and moments about the hip joint are shown in Fig. 1. Shortening the horizontal femoral offset (HFO) would reduce the range of abduction and the abductor muscle moment arm length (AMA), and therefore increase the muscle force required to counteract the moment about the joint arising from the body weight (BMF) in stance, and this would increase the resultant joint contact force - the opposite of the goal of positioning in total hip replacement surgery [56, 57]. Conversely, joint 'medialisation', or shortening the bodyweight moment arm (BMA) is a surgical goal because it results in a lower JCF by reducing the moment generated across the joint by the bodyweight. These effects were quantified in a biomechanical modelling study by Johnston et al [58], who predicted the reduction in JCF and AMF resulting from up to $20 \mathrm{~mm}$ joint centre medialisation and lateral trochanteric 
transfer (increasing HFO). Identical force magnitudes were applied to the models in this study, in order to isolate the structural effects of the prosthesis positioning. Similarly, any abductor muscle weakening from surgical incisions, or loss of soft tissue tension, was neglected. However, this is judged to be an acceptable simplification. Johnston et al's model [58] predicted that joint centre medialisation had a greater effect upon the joint force magnitudes than increasing the horizontal femoral offset. This may be extrapolated into reduction of HFO and AMA distances, and furthermore, Silva et al's study [49] reported that hip resurfacing resulted in a mean joint centre medialisation of $6.5 \mathrm{~mm}$, whereas the greatest reduction in abductor moment arm in this study was $4.2 \mathrm{~mm}$. With linear interpolation of Johnston et al's data [58], this medialisation would give a $12 \%$ lower JCF, compared to, at most, only a $3 \%$ increase in JCF as a result of the horizontal femoral offset increase. As such, the observed effects of the reduced femoral neck strains associated with a shorter joint horizontal femoral offset- improved femoral neck fracture strength but potentially an increased extent of stress shielding- are likely to become more marked if the associated joint contact and abductor muscle force reductions were taken into account. This study's models therefore represent a conservative case.

In the application of the joint contact force over a $5 \mathrm{~mm}$ radius circular patch, the Hertzian calculation was based on the initial, nominal clearance for the prosthesis design in question. The radius of the contact patch is dependent on the bearing clearance which is subject to manufacturing variability and will decrease on impacted implantation and as the prosthesis wears. The fact that a non-uniform pressure distribution was used, and that the stiffness of the prosthesis is considerably higher than that of the bone, support the assumption that within a feasible range, the contact patch diameter has a negligible effect upon the strain on the bone beneath the implant and cement mantle, so the nominal value was used.

A further simplification of the force application method was the use of a single, gait load case for the bone remodelling study. Particularly in a young hip resurfacing patient, a wide range of post-operative activities would be expected. However, Morlock et al [59] reported the activities of 31 total hip replacement patients of mean age 62.5 years, and their data suggested that normal walking may account for as much as $96 \%$ of a standard day's dynamic loading. Any more strenuous loading conditions would be discouraged by the surgeon in the immediately postoperative period, which this study aims to simulate. Considering the traumatic loading scenarios modelled, again only single load cases were modelled but 
these were designed to replicate in-vitro simplifications of, again, widely variable in-vivo traumatic load events [32, 35-37]. Worst case loading conditions from these studies were used, so it was considered that these load cases were a fair representation of common traumatic events.

\section{Conclusions}

Resurfacing hip replacement is an established treatment for osteoarthritis in the young, active patient, and excellent medium term results have been achieved. Occasional femoral neck fractures and femoral prosthesis loosenings occur, and this modelling study provides corroborative evidence to support the following predictions, in the understanding and mitigation of these failure modes:

1) following hip resurfacing, stress shielding can be contributed to narrowing of the femoral neck but only if there is a considerable reduction in the horizontal femoral offset. This was predicted to occur with valgus prosthesis positioning, if the prosthesis is undersized.

2) recreation of the natural horizontal offset, which is possible with an adequately sized femoral head prosthesis, considerably reduces the change from the natural to postoperative strain distribution.

3) the fracture strength of the intact femoral neck can be maintained after implantation of the resurfacing device provided the prosthesis is oriented in valgus relative to the femoral neck axis.

\section{ACKNOWLEDGMENTS}

This study was funded by a collaborative Knowledge Transfer Partnership between Finsbury Development

Ltd and the UK Government Technology Strategy Board, and was undertaken at the University of Southampton. 


\section{REFERENCES}

1. Amstutz, H.C., Clarke, I.C., Cristie, J., Graff-Radford, A., Total hip articular replacement by internal eccentric shells. Clin Ortho Rel Res, 1977. 128: p. 261-284.

2. Wagner, H., Surface replacement arthroplasty of the hip. Clin Ortho Rel Res, 1978. 134: p. $102-130$.

3. McMinn, D., Treacy, R., Lyn, K., Pynsent, P., Metal-on-metal surface replacement of the hip. Clin Ortho Rel Res, 1996. 329S: p. S89-S98.

4. Amstutz, H.C., Grigoris, P., Dorey, F.J., Evolution and future of surface replacement of the hip. J Ortho Sci, 1998. 3: p. 169-186.

5. Australian Orthopaedic Association National Joint Replacement Registry Annual Report. 2008, AOA: Adelaide.

6. Amstutz, H.C., Campbell, P.A., Le Duff, M.J., Fracture of the neck of the femur after surface arthroplasty of the hip. J Bone Joint Surg [Am], 2004. 86: p. 1874-1877.

7. Little, C.P., Ruiz, A.L., Harding, I.J., McLardy-Smith, P., Gundle, R., Murray, D.W., Athansou, N.A., Osteonecrosis in retrieved femoral heads after failed resurfacing arthroplasty of the hip. J Bone Joint Surg [Br], 2005. 87-B: p. 320-323.

8. Shimmin, A.J., Back, D., Femoral Neck Fractures Following Birmingham Hip Resurfacing: A National Review of 50 Cases. J Bone Joint Surg [Br], 2005. 87-B: p. 463-4.

9. Siebel, T., Maubach, S., Morlock, M.M., Lessons learned from early clinical experience and results of 300 ASR hip resurfacing implantations. Proc IMechE H, 2006. 220: p. 345353.

10. Treacy, R.B.C., McBryde, C.W., Pynsent, P.B., Birmingham hip resurfacing arthroplasty. J Bone Joint Surg [Br], 2005. 87-B: p. 167-170.

11. Beaulé, P.E., Campbell, P.A., Hoke, R., Dorey, F., Notching of the femoral neck during resurfacing arthroplasty of the hip. A vascular study. J Bone Joint Surg [Br], 2006. 88-B: p. 35-39.

12. Beaulé, P.E., Le Duff, M., Campbell, P., Dorey, F.J., Hyun Park, S., Amstutz, H.C., Metalon-metal surface arthroplasty with a cemented femoral component: a 7-10 year follow-up study. J Arthoplasty, 2004. 19(8): p. 17-22.

13. Hing, C.B., Young, D.A., Dalziel, R.E., Bailey, M., Back, D.L., Shimmin, A.J., Narrowing of the neck in resurfacing arthroplasty of the hip. J Bone Joint Surg [Br], 2007. 89-B: p. 1019-1024.

14. Pollard, T.C.B., Baker, R.P., Eastaugh-Waring, S.J., Bannister, G.C., Treatment of the Young Active Patient with Osteoarthritis of the Hip: A Five- to Seven-Year Comparison of Hybrid Total Hip Arthroplasty and Metal-on-Metal Resurfacing. J Bone Joint Surg [Br], 2006. 88-B: p. 592-600.

15. Spencer, S., Carter, R., Murray, H., Meek, R.M.D., Femoral Neck Narrowing After Metalon-Metal Hip Resurfacing. J Arthroplasty, 2008. 23: p. 1105-1109.

16. Huiskes, R., Chao, E.Y.S., A Survey of Finite Element Analysis in Orthopedic Biomechanics: the First Decade. J Biomech, 1983. 16: p. 385-409.

17. de Waal Malefijt, M.C., Huiskes, R., A clinical, radiological and biomechanical study of the TARA hip prosthesis. Acta Orthop Trauma Surg, 1993. 112: p. 220-225.

18. Wanatabe, Y., Shiba, N., Matsuo, S., Higuchi, F., Tagawa, Y., Inoue, A., Biomechanical study of the resurfacing hip arthroplasty- finite element analysis of the femoral component. J Arthoplasty, 2000. 15: p. 505-511.

19. Freeman, M.A.R., Some Anatomical and Mechanical Considerations Relevant to the Surface Replacement of the Femoral Head. Clin Ortho Rel Res, 1978. 134: p. 19-24.

20. Beaulé, P.E., Lee, J.L., Le Duff, M.J., Amstutz, H.C., Ebramzadeh, E., Orientation of the femoral component in surface arthroplasty of the hip. A biomechanical and clinical analysis. J Bone Joint Surg [Am], 2004. 86: p. 2015-2021.

21. Little, J.P., Taddei, F., Viceconti, M., Murray, D.W., Gill, H.S., Changes in Femur Stress after Hip Resurfacing Arthroplasty: Response to Physiological Loads. Clin Biomechanics, 2007. 22: p. 440-448. 
22. Taylor, M., Finite element analysis of the resurfaced femoral head. Proc IMechE H, 2006. 220: p. 289-297.

23. Gupta, S., New, A.M.R., Taylor, M., Bone remodelling inside a cemented resurfaced femoral head. Clin Biomechanics, 2006. 21: p. 594-602.

24. Ong, K.L., Kurtz, S.M., Manley, M.T., Rushton, N., Mohammed, N.A., Field, R.E., Biomechanics of the birmingham hip arthroplasty. J Bone Joint Surg [Br], 2006. 88-B: p. 1110-1115.

25. Long, J., Bartel, D.L., Surgical Variables Affect Mechanics of a Hip Resurfacing System. Clin Ortho Rel Res, 2006. 453: p. 115-122.

26. Radcliffe, I.A.J., Taylor, M., Investigation into the Effect of Varus-Valgus Orientation on Load Transfer in the Resurfaced Femoral Head: a Multi-Femur Finite Element Analysis. Clin Biomechanics, 2007. 22: p. 780-786.

27. Radcliffe, I.A.J., Taylor, M., Investigation into the Affect of Cementing Techniques on Load Transfer in the Resurfaced Femoral Head: A Multi-Femur Finite Element Analysis. Clin Biomechanics, 2007. 22: p. 422-430.

28. Ong, K.L., Day, J.S., Kurtz, S.M., Field, R.E., Manley, M.T., Role of Surgical Positioning on Interface Stress and Initial Bone Remodelling Stimulus around Hip Resurfacing Arthroplasty. J Arthroplasty, 2008. In Press.

29. Long, J., Santner, T.J., Bartel, D.L., Hip Resurfacing Increases Bone Strains Associated with Short-Term Femoral Neck Fracture. J Orth Res, 2009: p. 1-7.

30. Pal, B., Gupta, S., New, A.M., A Numerical Study of Failure Mechanisms in the Cemented Resurfaced Femur: Effects of Interface Characteristics and Bone Remodelling. Proc IMechE Part H: J Eng Med, 2009. 223: p. 471-484.

31. Morgan, E.F., Bayraktar, H.H., Keaveny, T.M., Trabecular Bone Modulus-Density Relationships Depend on Anatomic Site. J Biomech, 2003. 36: p. 897-904.

32. Cristofolini, L., Juszczyk, M., Martelli, S., Taddei, F., Viceconti, M., In Vitro Replication of Spontaneous Fractures of the Proximal Human Femur. J Biomech, 2007. 40: p. 28372845.

33. Hertz, H., On the contact of elastic solids. J Reine und Angewandte Mathematik, 1882. 92: p. 156-171.

34. Udofia, I.J., Yew, A., Jin, Z.M., Contact mechanics analysis of metal-on-metal hip resurfacing prostheses. Proc IMechE H, 2004. 218: p. 293-305.

35. Lotz, J.C., Hayes, W.C., The Use of Quantitative Computer Tomography to Estimate Risk of Fracture of the Hip from Falls. J Bone Joint Surg [Am], 1990. 72: p. 689-700.

36. Keyak, J.H., Rossi, S.A., Jones, K.A., Skinner, H.B., Prediction of femoral fracture load using automated finite element modelling. J Biomech, 1998. 31: p. 125-133.

37. Bessho, M., Ohnishi, I., Matsumoto, T., Ohashi., S., Tobita, K., Mastuyama, J., Nakamura, K., Prediction of Strength and Fracture Location of the Proximal Femur by a CT-based Nonlinear Finite Element Method- Effect of Load Direction on Hip Fracture Load and Fracture Site. Trans. Orthopaedic Research Society, 2008. 33: p. 955.

38. Bergmann, G., Deuretzbacher, G., Heller, M., Graichen, F., Rohlmann, A., Strauss, J., Duda, G.N., Hip contact forces and gait patterns from routine activities. J Biomech, 2001. 34: p. 859-871.

39. Keyak, J.H., Rossi, S.A., Prediction of femoral fracture load using finite element models: an examination of stress- and strain-based failure theories. J Biomech, 2000. 33: p. 209214.

40. Lotz, J.C., Cheal, E.J., Hayes, W.C., Fracture Prediction for the Proximal Femur using Finite Element Models: Part I- Linear Analysis. J Biomech Eng, 1991. 113: p. 353-360.

41. Lotz, J.C., Cheal, E.J., Hayes, W.C., Fracture Prediction for the Proximal Femur using Finite Element Models: Part II- Nonlinear Analysis. J Biomech Eng, 1991. 113: p. 361365.

42. Schileo, E., Taddei, F., Cristolfini, L., Viceconti, M., Subject-specific finite element models implementing a maximum principal strain criterion are able to estimate failure risk and fracture location on human femurs tested in-vitro. J Biomech, 2008. 41: p. 356-367. 
43. Bayraktar, H.H., Morgan, E.F., Niebur, G.L., Morris, G.E., Wong, E.K., Keaveny, T.M., Comparison of the Elastic and Yield Properties of Human Femoral Trabecular and Cortical Bone Tissue. J Biomech, 2004. 37: p. 27-35.

44. Weinans, H., Huiskes, R., Verdonschot, N., van Rietbergen, B., The Effect of Adaptive Bone Remodelling Threshold Levels on Resorption around Noncemented Hip Stems, in Advances in Bioengineering, R. Vanderby, Editor. 1991, ASME: New York. p. 303-306.

45. Weinans, H., Huiskes, R., van Rietbergen, B., Sumner, D.R., Turner, T.M., Galante, J.O., Adaptive bone remodelling around a bonded noncemented total hip arthroplasty: a comparison between animal experiments and computer simulation. J Orth Res, 1993. 11: p. 500-513.

46. Davis, E.T., Olsen, M., Zdero, R., Waddell, J.P., Semlitsch, E.H., Femoral Neck Fracture Following Resurfacing. J Bone Joint Surg [Br], 2008. 90-B: p. 1522-1527.

47. Morlock, M.M., Bishop, N., Zustin, J., Hahn, M., Ruther, W., Amling, M., Modes of Implant Failure after Hip Resurfacing: Morphological and Wear Analysis of 267 Retrieval Specimens. J Bone Joint Surg [Am], 2008. 90: p. 89-95.

48. Steffen, R.T., Pandit, H.P., Palan, J., Beard, D.J., Gundle, R., McLardy-Smith, P., Murray, D W, Gill, H.S., The five-year results of the Birmingham Hip Resurfacing Arthroplasty. J Bone Joint Surg [Br], 2008. 90-B: p. 436-441.

49. Silva, M., Haeng Lee, K., Heisel, C., dela Rosa, M.A., Schmalzried, T.P., The Biomechanical Results of Total Hip Resurfacing Arthroplasty. J Bone Joint Surg [Am], 2004. 86: p. 40-46.

50. Amstutz, H.C., Beaulé, P.E., Dorey, F.J., Le Duff, M.J., Campbell, P.A., Gruen, T.A., Metal-on-metal hybrid surface arthroplasty: two to six-year follow-up study. J Bone Joint Surg [Am], 2004. 86: p. 28-39.

51. Berend, M.E., Bertrand, T., Metal-Metal Hip Resurfacing: Solution to a Nonexistent Problem. Orthopedics, 2007. 30: p. 724-727.

52. Taylor, M.E., Tanner, K.E., Freeman, M.A.R., Yettram, A.L., Stress and Strain Distribution within the Intact Femur: Compression or Bending? Med Eng and Physics, 1998. 18: p. 122-131.

53. Aamodt, A., Lund-Larsen, J., Eine, J., Andersen, E., Benum, P., Schnell Husby, O., In Vivo Measurements Show Tensile Axial Strain in the Proximal Lateral Aspect of the Human Femur. J Orth Res, 1997. 15: p. 927-931.

54. Polgar, K., Gill, H.S., Viceconti, M., Murray, D.W., O'Connor, J. J., Strain Distribution within the Human Femur due to Physiological and Simplified Loading: Finite Element Analysis using the Standardized Femur Model. Proc IMechE Part H: J Eng Med, 2003. 217: p. 173-189.

55. Speirs, A.D., Heller, M.O., Duda, G.N., Taylor, W.R., Physiologically based boundary conditions in finite element modelling. J Biomech, 2007. 40: p. 2318-2323.

56. Charnley, J., Low Friction Arthroplasty of the Hip: Theory and Practice. 1979, New York: Springer.

57. McGrory, B.J., Morrey, B.F., Cahalan, T.D., An, K-N., Cabanela, M.E., Effect of Femoral Offset on Range of Motion and Abductor Muscle Strength after Total Hip Arthroplasty. J Bone Joint Surg [Br], 1995. 77-B: p. 865-869.

58. Johnston, R., Brand, R.A., Crowninshield, R.D., Reconstruction of the Hip: A Mathematical Approach to Determine Optimum Geometric Relationships. J Bone Joint Surg [Am], 1979. 61: p. 639-652.

59. Morlock, M.M., Schneider, E., Bluhm, A., Vollmer, M., Bergmann, G., Muller, V., Honl, M., Duration and Frequency of Every Day Activities in Total Hip Patients. J Biomech, 2001. 34: p. 873-881. 


\section{TABLES}

Table 1: Biomechanical Measurements for the Femur Resurfaced with a 50mm Prosthesis.

\begin{tabular}{|c|c|c|c|c|c|}
\hline & \multirow{2}{*}{\multicolumn{4}{|c|}{ Femur Model }} \\
\hline & & & & & \\
\hline & & Natural & $10^{\circ}$ Valgus & Neutral & $10^{\circ}$ Varus \\
\hline \multirow{3}{*}{$\begin{array}{r}\text { Horizontal Femoral Offset } \\
\text { (HFO) }\end{array}$} & Absolute / mm & 48.1 & 43.0 & 43.8 & 45.1 \\
\hline & vs. Natural / mm & - & -5.1 & -4.3 & -3.0 \\
\hline & $\%$ of Natural & - & $89 \%$ & $91 \%$ & $94 \%$ \\
\hline \multirow{3}{*}{$\begin{array}{r}\text { Abductor Moment Arm } \\
\text { (AMA) }\end{array}$} & Absolute / mm & 63.1 & 58.9 & 60.1 & 61.0 \\
\hline & vs. Natural / mm & - & -4.2 & -3.0 & -2.1 \\
\hline & $\%$ of Natural & - & $93 \%$ & $95 \%$ & $97 \%$ \\
\hline \multicolumn{2}{|c|}{ Mechanical Axis - Shaft Angle (MASA) $/^{\circ}$} & 5.9 & 5.28 & 5.38 & 5.54 \\
\hline
\end{tabular}




\section{FIGURES}

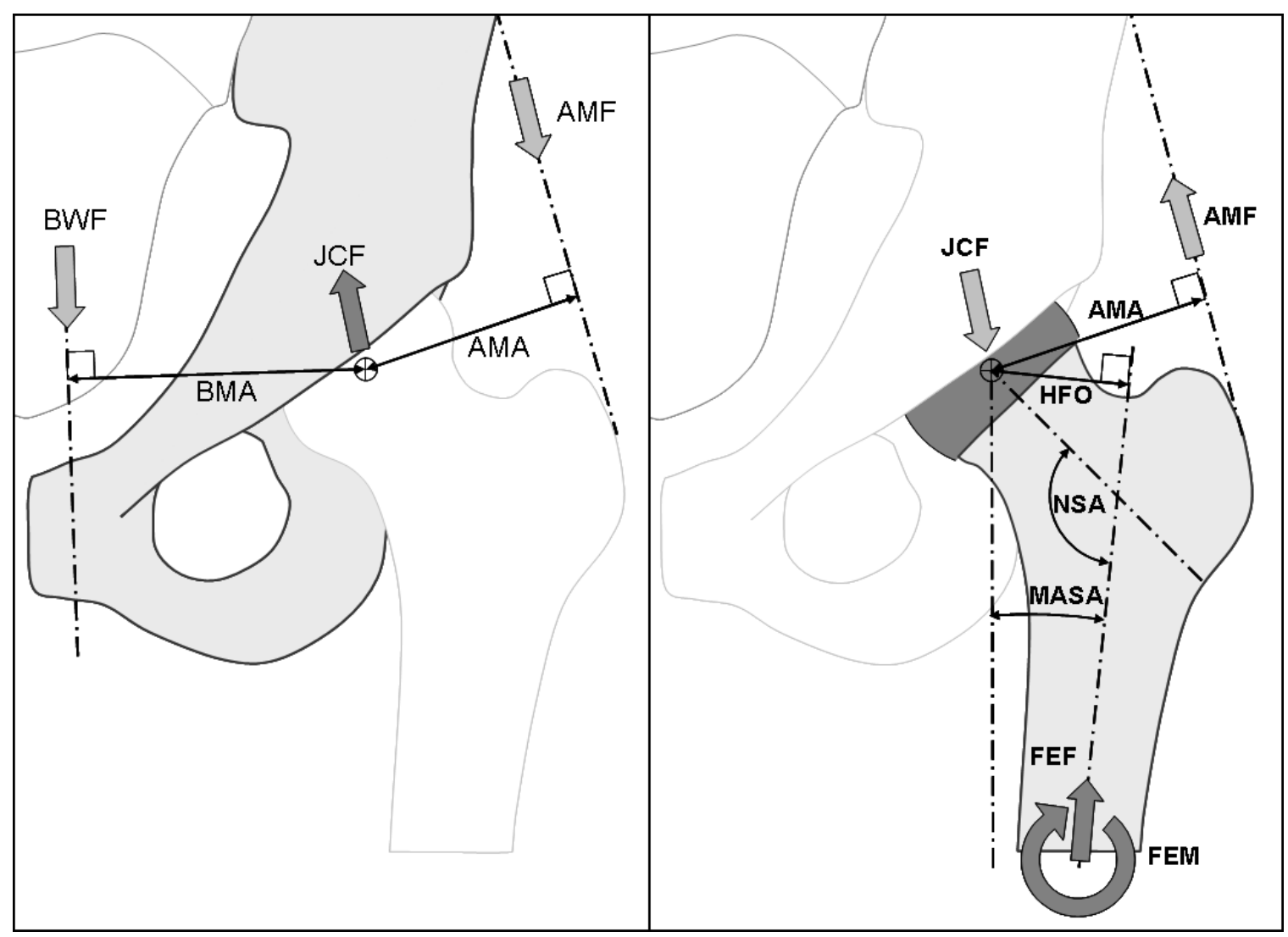

Fig. 1: Biomechanical Measurements and Forces on Hip.

Left: Pelvic Frame of Reference, Right: Femoral Frame of Reference. AMA=Abductor Moment Arm, AMF=Abductor Muscle Force, BMA=Bodyweight Moment Arm, BWF=Bodyweight Force, JCF=Joint Contact Force, FEF=Femur Equilibrium Force, FEM=Femur Equilibrium Moment, HFO=Horizontal Femoral Offset, MASA=Mech. Axis-Shaft Angle, NSA=Neck-Shaft Angle. 


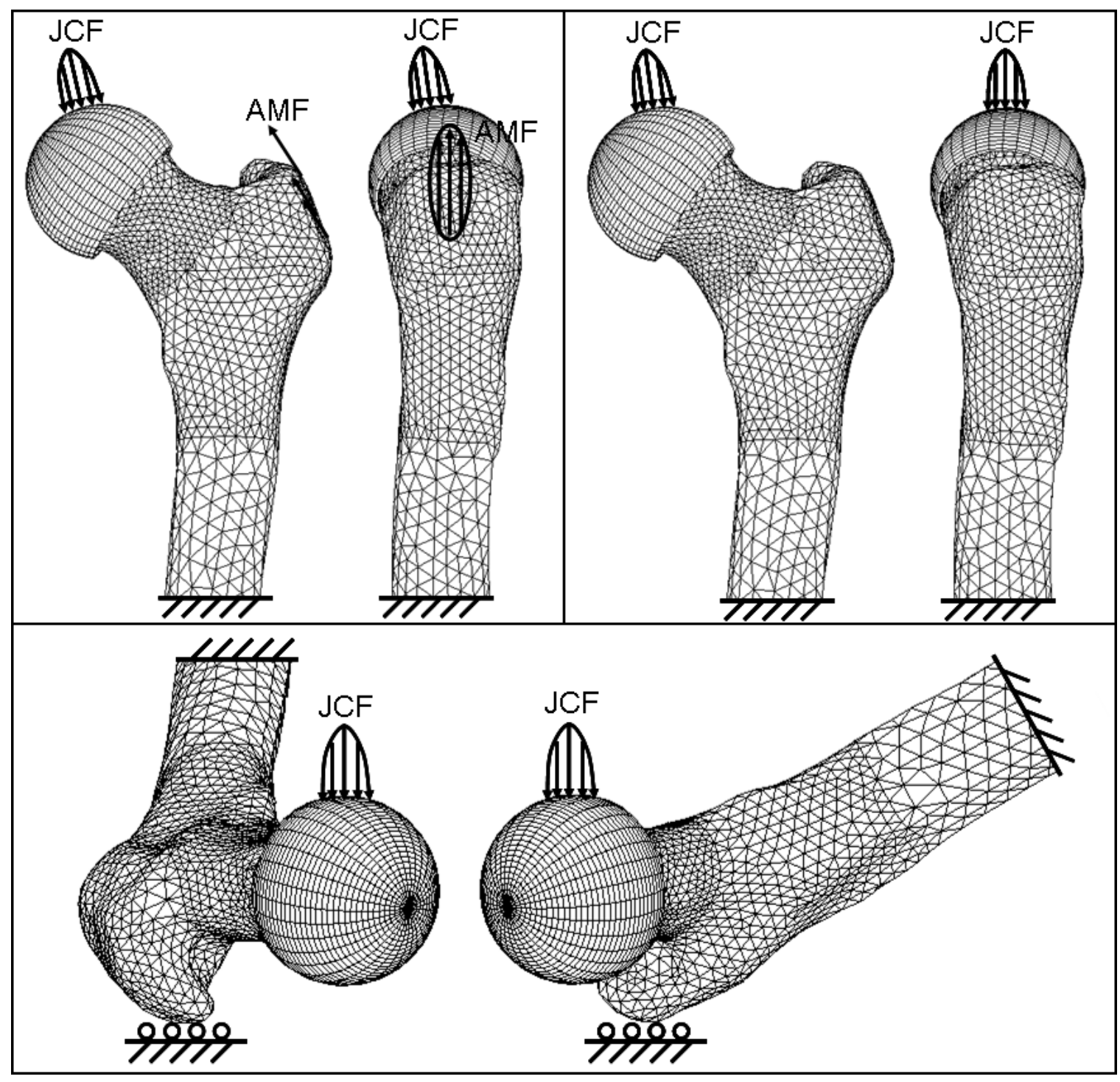

Fig. 2: Load Cases Investigated in the FE Modelling Study. Alignment and Boundary Conditions for: (top left) Gait, showing Joint Contact and Abductor Muscle Forces, (top right) Stumbling and (bottom) Sideways Falling. 


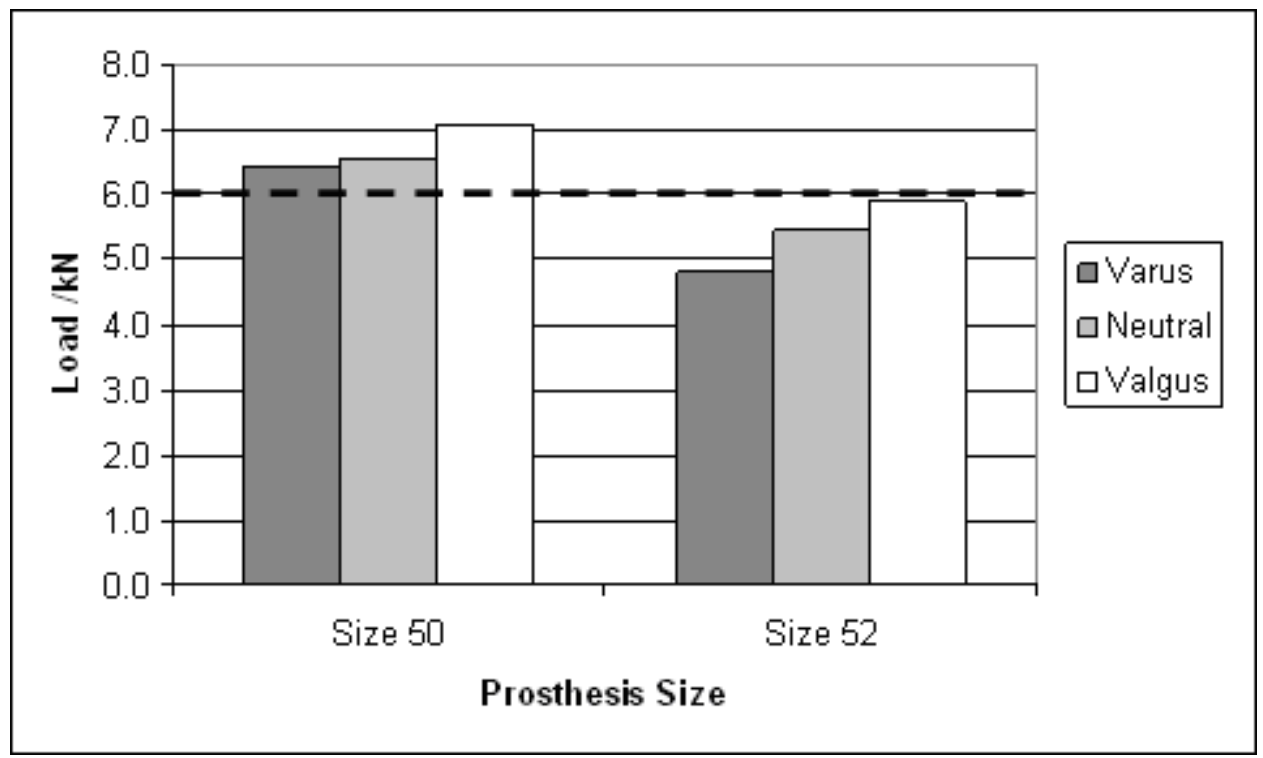

Fig. 3: Predicted Femoral Neck Fracture Load under Stumbling conditions, for the Femur Resurfaced with a 50mm Prosthesis (left) and a 52mm Prosthesis (right). Dashed Line Marks Fracture Load for Intact Bone

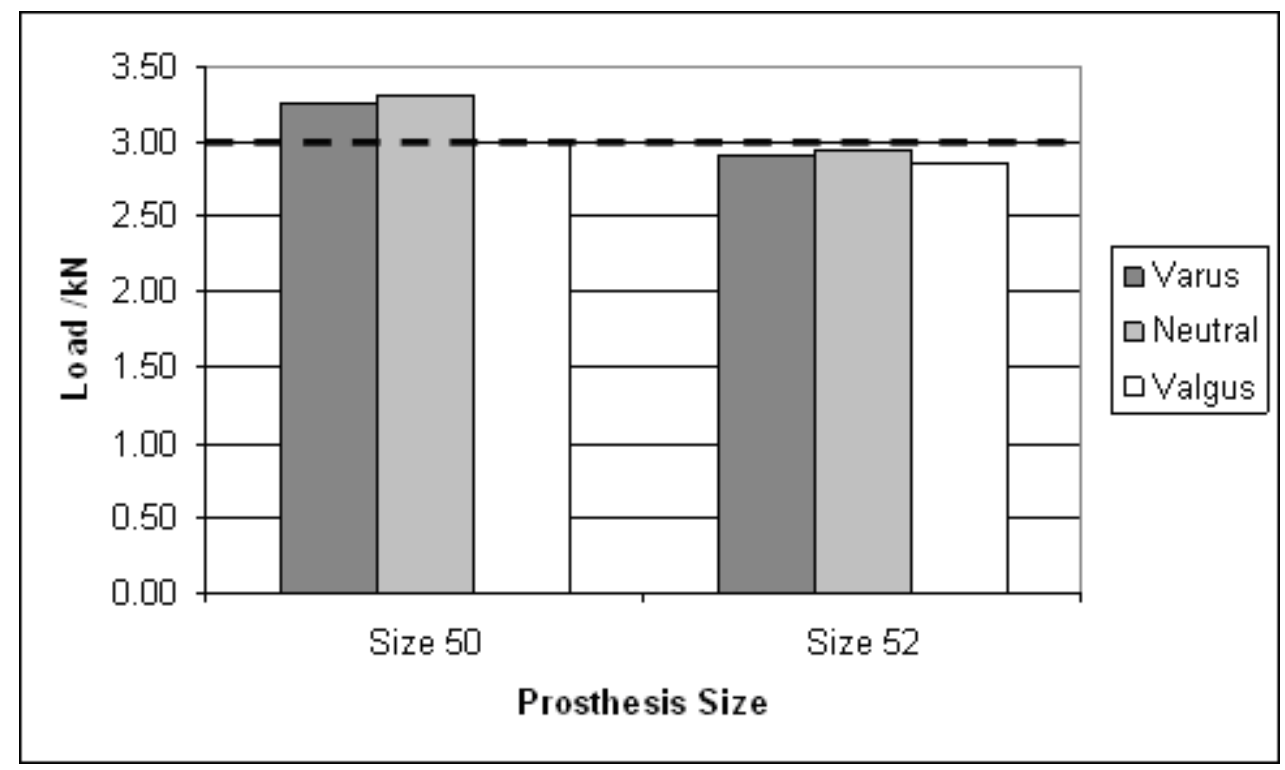

Fig. 4: Predicted Femoral Neck Fracture Load under Sideways Falling conditions, for the Femur Resurfaced with a 50mm Prosthesis (left) and a 52mm Prosthesis (right). Dashed Line Marks Fracture Load for Intact Bone 


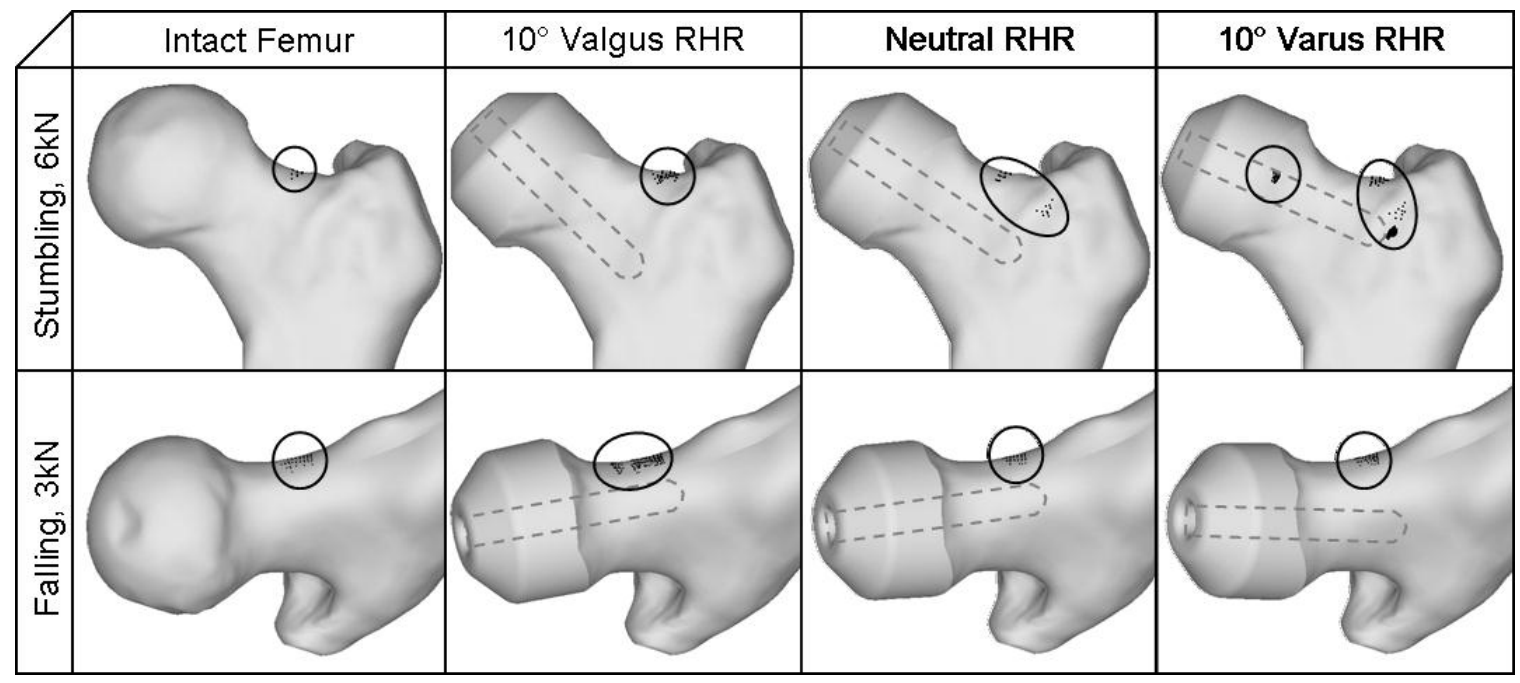

Fig. 5: Distribution of Yielding Bone Elements under Stumbling and Falling Loading Conditions, for the Natural Bone, and Resurfaced with the Prosthesis in Valgus, Neutral and Varus Orientation.

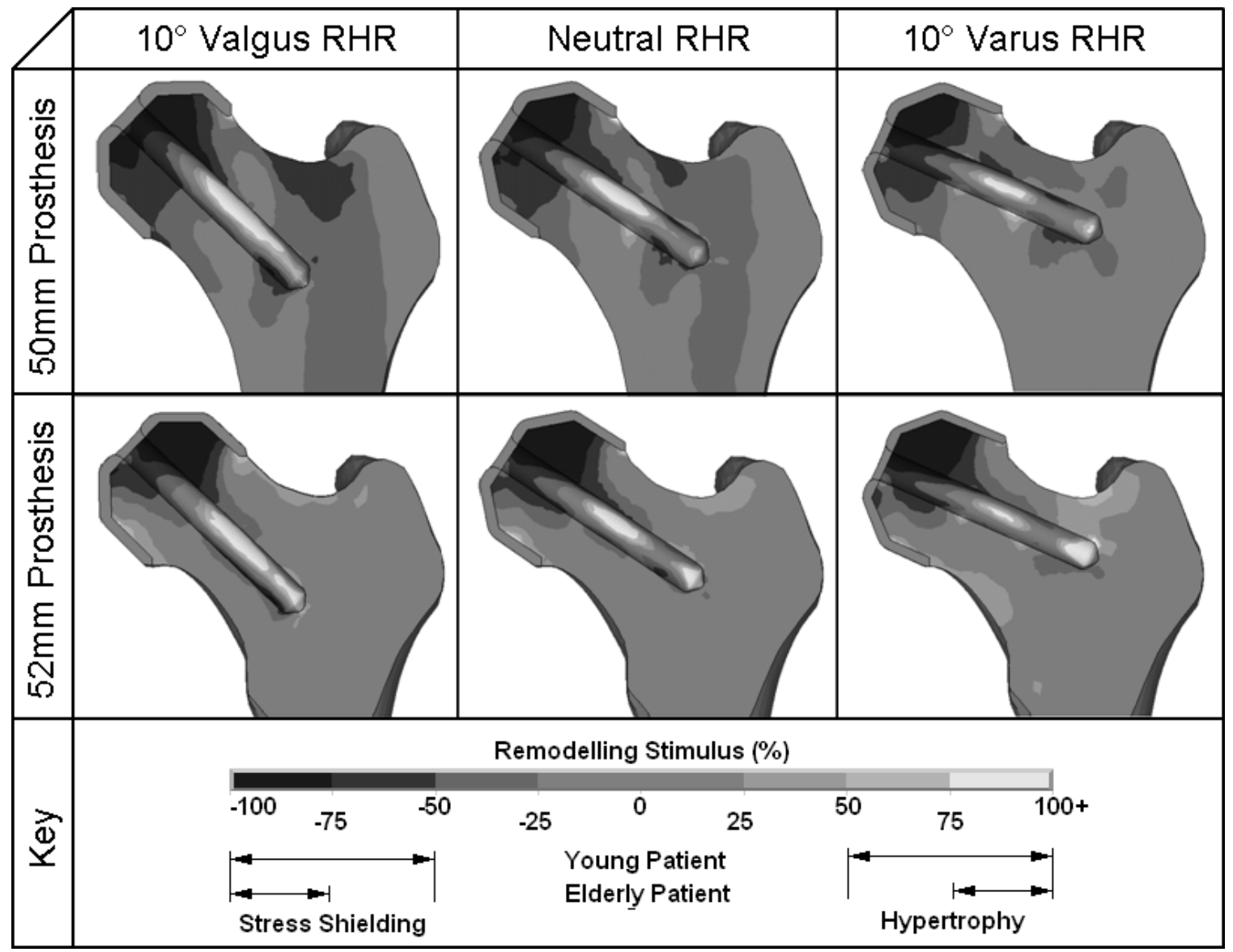

Fig. 6: Strain Energy Density Remodelling Stimulus for Resurfacing with Valgus (left), Neutral (middle) and Varus (right) oriented 50mm (top) and 52mm (bottom) Prostheses. 


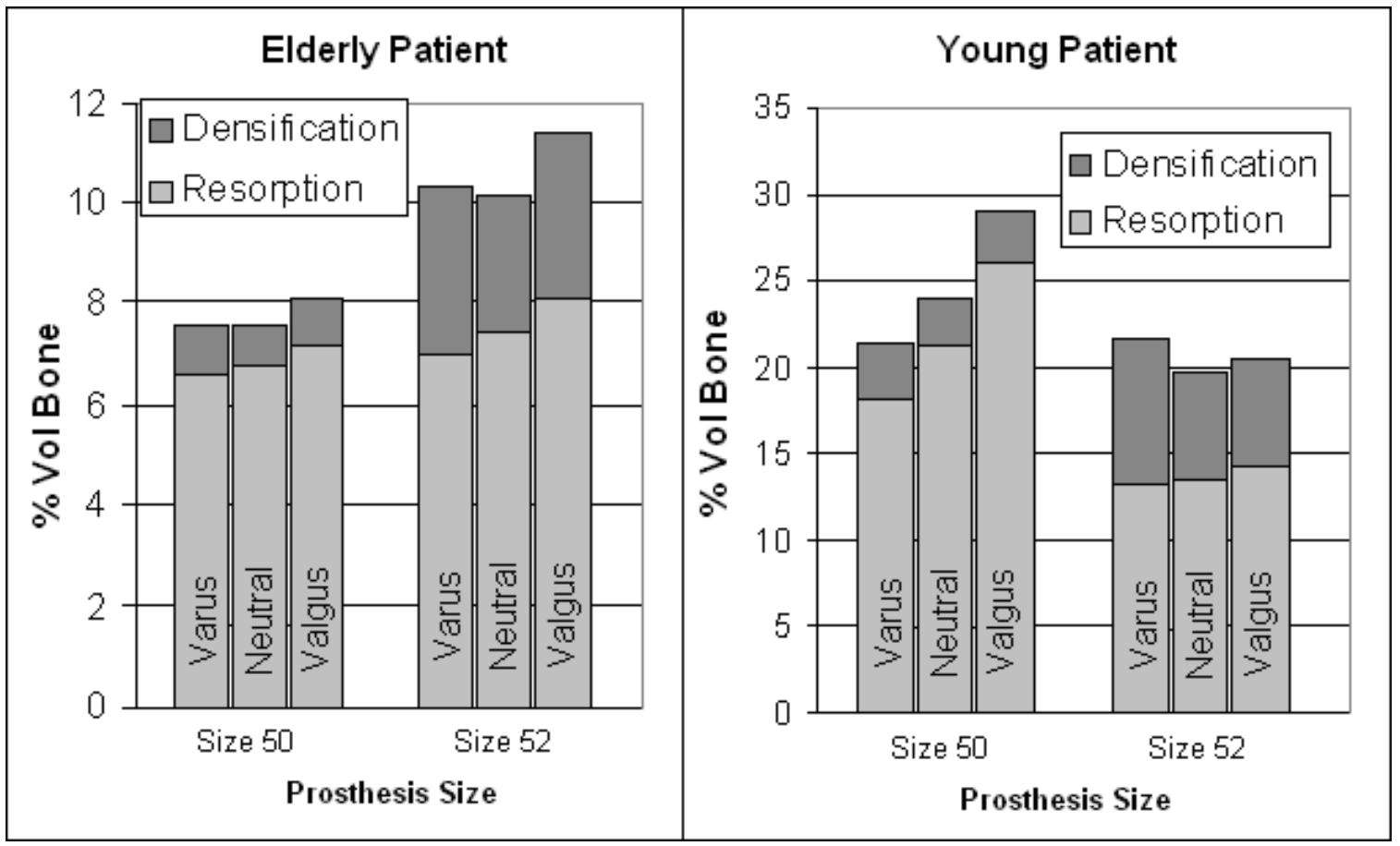

Fig. 7: Percentage Volumes of Remodelling Femoral Head and Neck Bone, Resurfaced with 50mm and 52mm Prostheses, in Elderly (left) and Young (right) Patients

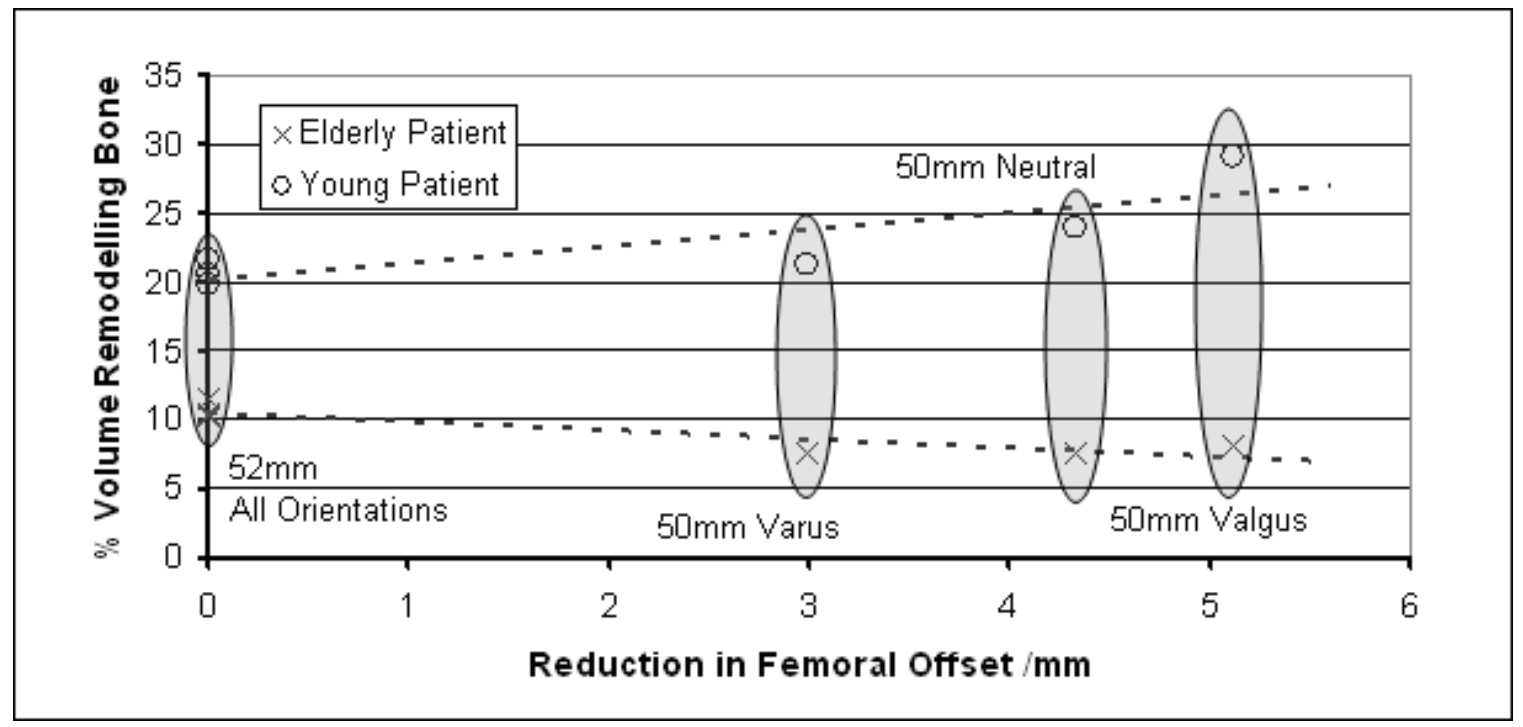

Fig. 8: Chart showing the Relationship between the Reduction in Horizontal Femoral Offset and the Volume of Remodelling Bone for Elderly and Young Patients. 


\section{APPENDIX: NOTATION}

$\begin{array}{ll}\text { AMA } & \text { Abductor Moment Arm, } \\ \text { AMF } & \text { Abductor Muscle Force, } \\ \text { BMA } & \text { Bodyweight Moment Arm, } \\ \text { BWF } & \text { Bodyweight Force, } \\ \text { JCF } & \text { Joint Contact Force, } \\ \text { FEF } & \text { Femur Equilibrium Force, } \\ \text { FEM } & \text { Femur Equilibrium Moment, } \\ \text { HFO } & \text { Horizontal Femoral Offset, } \\ \text { MASA } & \text { Mech. Axis-Shaft Angle, } \\ \text { NSA } & \text { Neck-Shaft Angle. }\end{array}$

